\title{
IMPACT OF DEPOLARIZATION EFFECTS ON MIMO POLARIZED WIRELESS CONFIGURATION
}

\author{
Kosai RAOOF, kosai.raoof@gipsa-lab.fr, Nuttapol PRAYONGPUN, prayongp@gipsa-lab.fr \\ Laboratoire Grenoble Images Paroles Signal Automatique (GIPSA-DIS), UMR CNRS 5216 \\ 961, Rue de la Houille Blanche - BP 46 - 38402 Saint Martin d'Hères, France
}

\begin{abstract}
On wireless communication systems, multiple-input multiple-output (MIMO) systems have dramatically the potential to improve the reliability and the performance. However, the MIMO wireless channel performance is generally affected by the antenna array configuration and environment characteristic. This paper highlights the impact of angle spread and depolarization effects on the MIMO channel capacity with a focus on $4 \times 4$ uniform linear multipolarized antenna array. We employ a geometric scattering model based on a three-dimensional double bouncing model that takes into account the antenna configurations. We investigate the use of multipolarization configurations that can provide capacity improvement over conventional single-polarization configurations. The single-polarization configuration is dependent on the depolarization phenomena. It is found that when low depolarization and spatial correlation effects, the capacity of single-polarization configuration behaves better than that of multipolarization configuration.
\end{abstract}

Index Terms - Multiple-input multiple-output (MIMO) channel capacity, spatial correlation, multipolarized antenna arrays, depolarization effects

\section{INTRODUCTION}

For next-generation wireless communication systems, multiple antennas at both transmitter and receiver could be engaged to achieve the higher capacity and reliability of wireless communication channels, under rich scattering environments, in comparisons with traditional single antennas. Due to the potential of MIMO systems on a limited bandwidth and transmission power, the initial researches demonstrate that the uncorrelated channel capacity can be proportionally increased according to the number of antennas. Unfortunately, in practice, the performances of MIMO communication channel are affected by spatial correlation and channel environments. The spatial correlation depends on the array configuration such as radiation pattern, antenna spacing and array geometry. The channel environments are dependent on the environment characteristics such as number of channel path, distribution and properties of scatterers, angle spread and cross-polarization discrimination. Thus, the antenna ar- rays at transmitter and receiver should be properly designed to reduce the spatial correlation effects and improve the communication performances.

However, it is possible to reduce this effect traditionally by increasing antenna array spacing [7] but it is not often suitable to apply in some wireless applications which limit the array size. Therefore, for eliminating the spatial correlation effects and remaining high transmission performances, there are essentially two diversity techniques such as pattern or angular and polarization diversity techniques [6],[8]. For pattern diversity technique, the radiation of antennas should be generated in manner to isolate the radiation pattern. For polarization diversity technique [6], the antennas are designed to radiate with orthogonal radiation polarizations to create uncorrelated channels across different array elements. In general, there are three diversity techniques employed on MIMO wireless system. However, there are also alternative techniques such as multimode diversity that exploits the difference of high order modes to obtain low correlated channel across the modes and a combination of pattern and polarization diversity techniques that take together the advantages of orthogonal radiation patterns and polarizations.

In this paper, we define a geometric scattering model based on a three-dimensional double bouncing model that takes into account the antenna configuration. All antennas are provided as a uniform linear array with isotropic antennas at transmitters and receivers. In the meantime, all scatterers are uniformly distributed on scattering areas while taking into account a cross-polar discrimincation (XPD). XPD indicates the ratio of the co-polarized average received power to the cross-polarized average received power. Therefore scattering matrix is used to describe the depolarization of incident wave from each scatterer. Afterward, to simplify the simulated environment configuration, we assume that the angle of arrival and that of departure are the uniform distribution for indoor situation.

We also present a simulation study of the spatial correlation and the channel capacity for single- and dualpolarized antenna arrays applied to $4 \times 4$ MIMO system while all of antenna elements are separated a half wavelength even in the case of the dual polarization configuration. In addition, we examine the cross-polar discrimination effects on MIMO polarized channel capacity for different antenna configuration. 


\section{ANTENNA ARRAY DESIGN}

For determining the multiple antenna transmission performances, not only the propagation environment has an important role but the proper implementation of the antennas plays also another dominant role in practice. For expample, some classic antenna array geometries are uniform linear arrays, uniform circular arrays or uniform rectangular arrays. Consequently, due to the different array configurations, their correlation effect is produced in a different way.

The goal of pattern and polarization diversities is to decrease the correlation effect between antenna elements. In this paper, using multiple polarization technique is a reasonable alternative in indoor room and some environments for which the depolarization is high. Therefore our current interest is focused on azimuth and elevation isotropic antennas applied to a uniform linear array wireless system.

\begin{tabular}{|c|c|c|}
\cline { 2 - 3 } \multicolumn{1}{c|}{} & $G_{\theta}$ & $G_{\phi}$ \\
\hline$G_{\theta}(\theta, \phi)$ & 1 & 0 \\
\hline$G_{\phi}(\theta, \phi)$ & 0 & 1 \\
\hline
\end{tabular}

Table 1: Patterns for different electric dipoles

We consider that different radiating patterns of the antennas are in the far field case simplified by neglecting pathloss and distance phase. These radiating patterns are simply dependent on the azimuth and elevation angle direction as shown in table 1 , where a general expression of radiation patterns are given by [1]

$$
G=G_{\theta}(\theta, \phi) \vec{\theta}+G_{\phi}(\theta, \phi) \vec{\phi}
$$

where $G_{\theta}(\theta, \phi)$ and $G_{\phi}(\theta, \phi)$ are the amplitudes of polarization vector at elevation and azimuth direction.

\section{GEOMETRIC SCATTERING MODELLING}

We focus on a useful model, geometric scattering modelling which is based on an assumption that scatterers around the transmitter and receiver organize the AOD and AOA respectively within transmit and receive scattering areas. The scatterers are randomly located with according to a certain probability distribution. In particular, the scatterers are additionally used to represent the depolarization and attenuation mechanism of incident wave. To reduce the computational time, one propagation path channel occur when one of transmit and one of receive scatterers are randomly linked. Then the actual channel impulse response is then established by a simplified ray-tracing route.

By using our simulated double bounce geometric scattering model as seen in Fig.1, we employ a uniform linear array at both transmitter and receiver. The height of transmitter and receiver has the same level. Moreover, transmit and receive scatterers are uniformly distributed within an angular region characterized by $|\phi+\pi / 2| \leq \Delta \phi / 2$ in elevation area and $|\theta+\pi / 2| \leq \Delta \theta / 2$ in azimuth area at transmitter and $|\phi-\pi / 2| \leq \Delta \phi / 2$ in elevation area and $|\theta-\pi / 2| \leq \Delta \theta / 2$ in azimuth area at receiver.

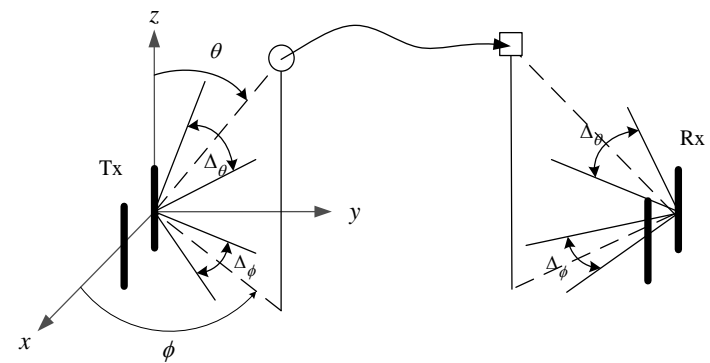

Fig. 1: Geometries of MIMO channel

Subsequently transmit and receive scatterers are randomly paired as previously mentioned. From one transmit scatterer to one receive scatterer for determining one propagation path, there is a double depolarization mechanism which is replaced by one scattering matrix. We also assume that the channel coherence bandwidth is larger than the transmitted bandwidth of the signal. This channel is usually called frequency non-selective or flat fading channel.

In case of far field transmission, the narrowband (flat fading) transmission channel between the antenna $p$ at the transmitter and the antenna $m$ at the receiver can be expressed as

$$
\begin{aligned}
h_{m p}(t, f)= & \frac{1}{\sqrt{N_{S}}} \sum_{i=1}^{N_{S}} a_{m}^{(i)} a_{p}^{(i)} \exp \left\{-j \vec{k}^{(i)} \cdot \vec{v}_{R x} t-j \vec{k}^{\prime(i)} \cdot \vec{v}_{T x} t+\varphi_{m p}\right\} \\
& {\left[G_{\theta}^{m}\left(\theta_{i}, \phi_{i}\right) G_{\phi}^{m}\left(\theta_{i}, \phi_{i}\right)\right] S_{m p}^{(i)}\left[\begin{array}{c}
G_{\theta}^{p}\left(\theta_{i}, \phi_{i}\right) \\
G_{\phi}^{p}\left(\theta_{i}, \phi_{i}\right)
\end{array}\right] }
\end{aligned}
$$

where $N_{S}$ is the number of scatterers at the receiver and the transmitter; $\vec{v}_{T x}$ and $\vec{v}_{R x}$ are the velocity vector of the transmitter and the receiver; $\vec{k}^{\prime(i)}$ and $\vec{k}^{(i)}$ are the vectors of wave number in the direction of the $i$ th transmit scatterer and the $i$ th receive scatterer where $\left|\vec{k}^{(i)}\right|=\left|\vec{k}^{\prime(i)}\right|=2 \pi / \lambda ; G_{\theta}^{p}\left(\theta_{i}, \phi_{i}\right)$ and $G_{\phi}^{p}\left(\theta_{i}, \phi_{i}\right)$ are the gain in the direction of $\vec{\theta}$ and $\vec{\phi}$ of the $p$ th transmit antenna in the direction of the $i$ th transmit scatterer. $G_{\theta}^{m}\left(\theta_{i}, \phi_{i}\right)$ and $G_{\phi}^{m}\left(\theta_{i}, \phi_{i}\right)$ are the gain in the direction $\vec{\theta}$ and $\vec{\phi}$ of the $m$ th receive antenna in the direction of the $i$ th receive scatterer; $t$ is time; $a_{m}^{(i)}$ is the $m$ th element of the local vector of the receive antenna, so that the local receive vector can be expressed as $\mathbf{a}_{\mathrm{Rx}}^{(i)}=\left[\begin{array}{llll}1 & e^{-j \vec{k}^{(i)} \cdot \vec{r}_{1}} & \cdots & e^{-j \vec{k}^{(i)} \cdot \vec{r}_{M-1}}\end{array}\right], a_{p}^{(i)}$ is the $p$ th element of the local vector of the transmit antenna, where a local transmit vector is expressed as $\mathbf{a}_{\mathrm{Tx}}^{(i)}=\left[\begin{array}{llll}1 & e^{-j k^{\prime(i)} \cdot \vec{r}_{1}^{\prime}} & \cdots & e^{-j k^{(i)} \cdot \vec{r}_{N-1}^{\prime}}\end{array}\right] ; \mathbf{S}_{m p}^{(i)}$ are the scattering matrix for the $i$ th transmit scatterer and the $i$ th receive scatterer. We consider that $\mathbf{S}_{m p}^{(i)}$ is a $2 \times 2$ matrix contain- 
ing the random coefficients of the $i=1, \ldots, N_{S}$ wave components given by

$$
\mathbf{S}_{m p}^{(i)}=\left[\begin{array}{ll}
\sqrt{\frac{\mathrm{xpd}_{1}}{1+\mathrm{xpd}_{1}}} S_{\theta \theta}^{(i)} & \sqrt{\frac{1}{1+\mathrm{xpd}_{2}}} S_{\phi \theta}^{(i)} \\
\sqrt{\frac{1}{1+\mathrm{xpd}_{1}}} S_{\theta \phi}^{(i)} & \sqrt{\frac{\mathrm{xpd}_{2}}{1+\mathrm{xpd}_{2}}} S_{\phi \phi}^{(i)}
\end{array}\right]
$$

where the $S$ terms are defined as independent identically distributed (i.i.d) complex Gaussian and xpd denote the ratio of the co-polarized average received power to the cross-polarized average received power.

\section{MIMO CAPACITIES}

The advantages of antenna polarization diversity are very helpful on MIMO systems for reducing the antenna array size and the spatial correlation in order to obtain better capacity. With these advantages, the multi-polarized antennas become more and more interesting in MIMO transmission. Thus, in this paper, azimuth and elevation isotropic antennas are employed to investigate the potential of using the multi-polarized antennas as explained in previous section. Mean or ergodic capacity is employed to demonstrate the MIMO channel performances with $N$ transmit and $M$ receive antennas. The channel is perfectly known to the receiver but unknown to the transmitter. At each transmit antenna, the transmitted signals have the same power. The ergodic channel capacity, taken over the probability distribution of transmission channel matrix $\mathbf{H}$, can be exactly calculated via Monte Carlo simulations, is given by [3]

$$
C_{E r g}=\mathrm{E}_{\mathbf{H}}\left[\log _{2} \operatorname{det}\left(\mathbf{I}_{M}+\frac{\rho}{N} \mathbf{H} \mathbf{H}^{H}\right)\right]
$$

where $M$ and $N$ denotes the number of antennas at receivers and transmitters. $\mathbf{H}$ is the transmission channel matrix and $\rho$ is signal-to-noise ratio. The transmission channel matrix $\mathbf{H}$ is naturally correlated by transmit and receive antenna arrays.

\section{SIMULATIONS AND RESULTS}

\subsection{Capacity Versus Angle Spread}

The antenna correlation effect is actually one important indicator of transmission performances since lower correlation will tend to produce higher mean channel capacity for single polarization system as seen in Fig.2. Thus employing the polarization and angular diversity techniques are attractive way to improve MIMO systems. The $4 \times 4$ MIMO systems employ isotropic antennas for $\lambda / 2$ antenna spacing as shown in Fig.1. In orders to estimate the channel capacity of different antenna configuration, the simulated environments must be identical. Then the channel capacities are studied in terms of different antenna configurations. The radiation patterns of each antenna are normalized by the radiation pattern of an isotropic antenna.

As mentioned in previous section, the distribution of angles of departure is assumed to have a uniform elevation distribution $|\phi+\pi / 2| \leq \Delta \phi / 2$ and a uniform arrival azimuth distribution $|\theta+\pi / 2| \leq \Delta \theta / 2$ and the distribution of angles of arrival is assumed to have a uniform elevation distribution $|\phi-\pi / 2| \leq \Delta \phi / 2$ and a uniform arrival azimuth distribution $|\theta-\pi / 2| \leq \Delta \theta / 2$ where $\Delta \phi=\Delta \theta=\mathrm{AS}$ and $\mathrm{xpd}_{1}=\mathrm{xpd}_{2}=0 \mathrm{~dB}$ with 20 scatterers at both transmitter and receiver and $15 \mathrm{~dB}$ SNR. The aim of this section is to study the effects of angle spreads and antenna radiation patterns in terms of ergodic capacity.

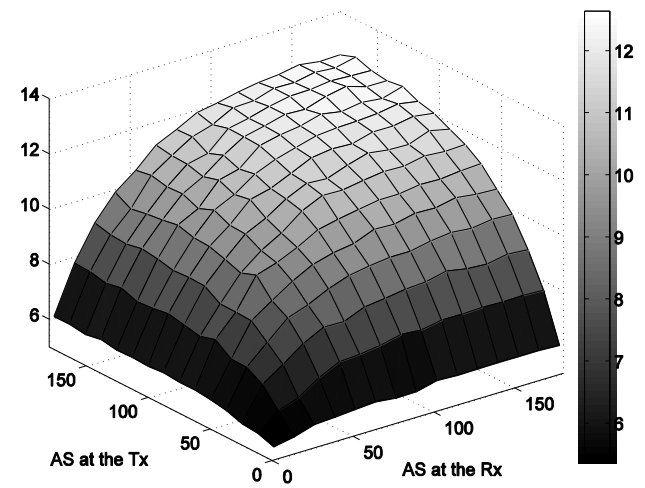

(a)

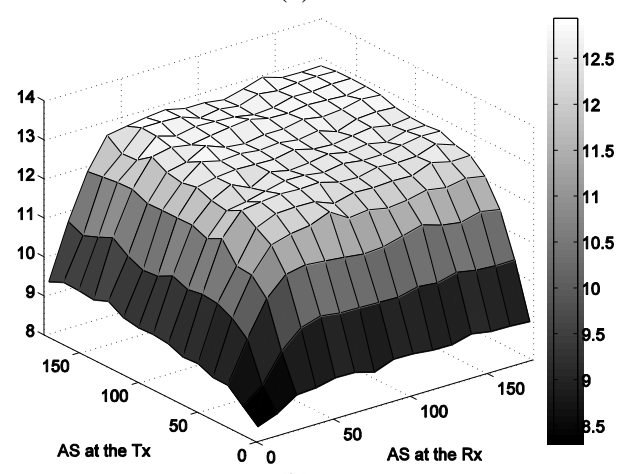

(b)

Fig. 2: 4×4 MIMO channel capacity of isotropic antennas: (a) single-polarization system and (b) dual-polarization system

Fig. 2 demontrates the $4 \times 4$ MIMO channel capacity of isotropic antennas with single-polarization in Fig.2a and with dual-polarization in Fig.2b. For single-polarization case, only azimuth isotropic antennas are employed and for dual-polarization case, we put successively azimuth and elevation isotropic antennas in order with $\lambda / 2$ antenna spacing. From Fig.2a, the MIMO channel capacity increases as the angle spread increases at transmitter and receiver for the same polarization antennas. In contrast, the dual polarization achieves better channel capacity due to the lower antenna correlation. It found that the MIMO channel capacity is significantly dependent on the antenna correlation. The polarization diversity technique can diminish the spatial correlation effect and improve the system performances as shown in Fig.2b. 


\subsection{Capacity versus Depolarization Effects}

While the multi-polarized antenna array is employed, the spatial correlation effect can be reduced or eliminated due to low radiation pattern interference. Nevertheless, the cross-polarization discrimination (XPD) becomes the most important parameter because XPD represents the ratio of the co-polarized average received power to the cross-polarized average received power. Then, with high XPD value, the less energy is coupled between the crosspolarized channels. Even if the capacity of multipolarized antenna arrays can remain high particularly at lower XPD and the higher K-factor values [9], singlepolarized antenna array performance can effectively provide better than that of multi-polarized antenna array at higher XPD and lower spatial correlation value.

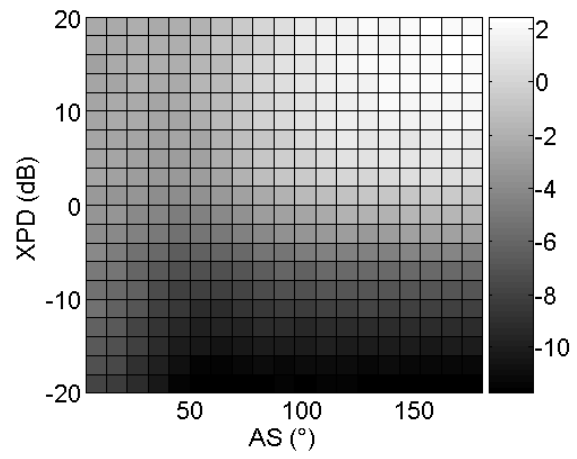

Fig. 3: Difference between the triple-polarized and the single-polarized channel capacity of $4 \times 4$ MIMO systems in the functions of XPD and AS

Fig. 3 explains difference between the triple-polarized and the single-polarized channel capacity of $4 \times 4$ MIMO systems $\left(\Delta C=C_{\text {single-polar }}-C_{\text {dual-polar }}\right) \mathrm{n}$ the functions of XPD and AS. We also consider that they have the same angle spreads (AS) at transmitter and receiver. For a high XPD and a sufficiently large angle spread, we note that the MIMO channel capacity of the single-polarized antenna is superior to that of the dual-polarized antenna because a product of the subchannel power is higher. By utilizing the Frobenius norm, it confirms that for higher XPD, the average transmission power of single-polarized isotropic antenna arrays is $\|\mathbf{H}\|_{F}=M N$. While lower XPD, the average transmission power of single-polarized isotropic antenna arrays tends to zero, $\|\mathbf{H}\|_{F} \Rightarrow 0$, because of the mismatch of polarization. In contrast, the average transmission power of dual-polarized isotropic antenna arrays is always equal to $\|\mathbf{H}\|_{F} \approx M^{\theta} N^{\theta}+M^{\phi} N^{\phi}$ for higher XPD and $\|\mathbf{H}\|_{F} \approx M^{\phi} N^{\theta}+M^{\theta} N^{\phi}$ for lowers XPD.

\section{CONCLUSION}

The performance of MIMO communication systems is essentially affected by the spatial correlation and channel environments. The spatial correlation depends on the ar- ray configurations and the channel characteristics. Therefore to achieve the optimum performances on MIMO systems, the proper selection of array configuration is required. In this paper, we studied the MIMO wireless channel capacity of single- and multi-polarized antenna arrays applied to a uniform linear array with two isotropic antenna configurations.

The simulation results demonstrate that for the nonline-of-sight (NLOS) case, the use of multi-polarization antennas can provide capacity improvement over conventional single-polarization antennas for narrow angle spread. However, when the cross-polarization discrimination is superior than $0 \mathrm{~dB}$ corresponding to high copolarized channel power and low cross-polarized channel power, the subchannel power of single-polarization system can be higher by employing the same polarization as that of the co-polarized channel. Thus, with high XPD and low spatial correlation values, single-polarized antenna array performance can effectively provide better capacity than that of multi-polarized antenna array. Finally, the cross-polarization discrimination should be also investigated before employing the polarization diversity technique.

\section{REFERENCES}

[1] Constantine A. Balanis, "Antenna Theory," second Edition, John Wiley \& Sons, New York, 1997.

[2] L.M. Correia, Ed., Wireless Flexible Personalised Communication (COST 259 Final Report), Wiley,2001

[3] G.J. Foschini and M.J. Gans, "On the limits of wireless communications in a fading environment when using multiple antennas," Wireless Personal communication., vol. 6 ,pp. 311335, Mar.1998.

[4] D.-S. Shiu,G. J. Foschini and M.J. Gans, "Fading Correlation and Its Effect on the Capacity of Multielement Antenna Systems," IEEE Trans. Comm.,vol. 48,no. 3,pp. 502513, Mar. 2000.

[5] M. Ali Khalighi, K. Raoof and G. Jourdain, "Capacity of Wireless Communication Systems Employing Antenna Arrays, a Tutorial Study," Kluwer Academic Publishers, vol. 23, no. 23, pp. 321-352, 2002.

[6] M.R. Andrews, P.P. Mitra, and R. deCarvalho, "Tripling the capacity of wireless communication using electromagnetic polarization," Nature, vol. 409, pp. 316-318, Jan. 2001.

[7] M.A. Jensen and J.W. Wallace, "A Review of Antennas and Propagation for MIMO Wireless Communication," IEEE Trans. Antennas Propagat., vol.52 No. 11, Nov. 2004.

[8] C. Oestges, V. Erceg and A.J. Paulraj, " "Propagation Modeling of MIMO Multipolarized Fiwed Wireless Channels," IEEE Trans. Veh. Technol., vol. 53, no. 3,May 2004.

[9] N. Prayongpun and K. Raoof, "MIMO Channel Capacities in Presence of Polarization Diversity with and without Line-ofSight Path," Journal WSEAS Trans. on Commun., vol. 5, no. 9, pp. 1744-1750, Sep. 2006.

[10] K. Raoof and N. Prayongpun, "Channel Capacity Performance for MIMO polarized diversity systems," IEEE/WCNM2005, pp. 1-4, Sep. 23-26, 2005. 